\title{
Respuesta de diatomeas a eventos de incendios en las cuencas de dos lagos de Patagonia Norte, Chile: Análisis del registro sedimentario
}

\author{
Rubí Mendoza ${ }^{1}$, Fabiola Cruces ${ }^{1}$, Denisse Álvarez ${ }^{2,3, *}$, Fernando Torrejón ${ }^{2}$, Alberto Araneda ${ }^{2}$ \\ y Roberto Urrutia ${ }^{2}$
}

${ }^{1}$ Departamento de Botánica, Facultad de Ciencias Naturales y Oceanográficas. Universidad de Concepción. Concepción, Chile.

${ }^{2}$ Facultad de Ciencias Ambientales, Universidad de Concepción. Concepción. Chile.

${ }^{3}$ Facultad de Ciencias, Universidad Santo Tomás. Concepción. Chile.

* Autor responsable de la correspondencia:dealvarez@udec.cl

Recibido: 18/08/2014

Aceptado: 19/05/2015

\section{RESUMEN}

Respuesta de diatomeas a eventos de incendios en las cuencas de dos lagos de Patagonia Norte, Chile: Análisis del registro sedimentario

Los incendios forestales pueden generar alteraciones en los ecosistemas acuáticos, debido principalmente al aumento de nutrientes por un incremento en la erosión y escorrentía. Sin embargo, se han observado resultados contradictorios en la respuesta biológica de estos ecosistemas, dependiendo de las condiciones ambientales de cada región. Por lo anterior, el objetivo del presente trabajo, fue evaluar mediante un estudio paleolimnológico, la respuesta de las comunidades de diatomeas, presentes en los lagos Thompson y Frío, ubicados en la Región de Aysén (Patagonia Norte), a los incendios forestales como consecuencia de la colonización de la zona. Los resultados permiten indicar que el lago Thompson presentó cambios significativos en la composición de las asociaciones de diatomeas, con una recuperación posterior al periodo de incendios. Sin embargo, el lago Frío no mostró cambios significativos en respuesta al periodo de incendios, ni en la composición específica de diatomeas, ni en parámetros comunitarios tales como abundancia absoluta, riqueza, diversidad, dominancia y razón diatomeas planctónicas/bentónicas. Estas diferencias entre ambos lagos podrían ser atribuidas, en orden de importancia, al estado trófico existente antes de la ocurrencia de los incendios, la pendiente de la cuenca y el volumen del lago.

Palabras clave: Diatomeas, sedimento, incendios, Patagonia Norte.

\begin{abstract}
Response of diatom to fire events in watersheds of two Northern Patagonian lakes, Chile: Analysis of the sedimentary record

Forest fires can generate changes in aquatic ecosystems, mainly due to increased nutrients by increased erosion and runoff. However, contradictory results were observed in the biological response of these ecosystems, depending on the environmental conditions in each region. The aim of this study was to asses, using a paleolimnological study, the response of diatoms communities in Thompson and Frío lakes located in the Region of Aysén (Northern Patagonian) to wildfires as a consequence of colonization of the area. The results indicated that the Thompson Lake presented significant changes in the composition of diatom assemblages due to fire and a post-fire recovery period. However, the Frío Lake showed no significant changes in response to the fire period, neither in diatom species composition nor in community parameters such as the absolute abundance, richness, diversity, dominance and the ratio between planktonic/benthonic diatoms. Such differences between lakes could be attributed, in order of importance, to the trophic status existing before the occurrence of fires, the watershed slope and the lake volume.
\end{abstract}

Key words: Diatom, sediment, fires, Northern Patagonian. 


\section{INTRODUCCIÓN}

Las actividades resultantes del asentamiento humano tales como la deforestación, incendios de bosques y cambios en el uso del suelo, han generado perturbaciones en los ecosistemas acuáticos (Watchorn et al., 2008; Theissen et al., 2012).

Los incendios forestales tienen un impacto similar al de la deforestación (Bergeron et al., 2000), considerándose como una de las perturbaciones más abruptas e importantes que pueden ocurrir en una cuenca (Whitlock, 2001). El efecto directo de éstos es la pérdida de la vegetación nativa que genera un aumento de la escorrentía, lo que a su vez promueve la entrada de sedimentos al cuerpo de agua (Markgraf et al., 2007). Entre los efectos indirectos se encuentran los cambios en la calidad de agua, asociados con una alta entrada de nutrientes, lo que puede conducir a la eutrofización del lago y generar efectos deletéreos sobre los ecosistemas acuáticos como el aumento de la producción primaria total, cambios en los ciclos biogeoquímicos y comunidades biológicas (Schelske et al., 1999; Philibert et al., 2003b; Smith et al., 2006; McWethy et al., 2010).

En los ecosistemas acuáticos las comunidades de algas, y en particular las poblaciones de diatomeas, constituyen un grupo diverso, que se establece en un amplio rango de hábitats. Su distribución está muy relacionada con la química del agua, la estacionalidad y la geología de la cuenca (Battarbee, 1986). Su rápido crecimiento permite que respondan rápidamente tanto a la eutrofización como a la recuperación del sistema (Alefs \& Müller, 1999; Anderson et al., 2005).

Estudios que relacionan cambios en asociaciones de diatomeas producto de los asentamientos humanos han documentado que, a través del periodo de asentamiento y despeje del paisaje, la concentración y diversidad de diatomeas aumenta significativamente en un corto periodo temporal (Langman, 1971; Garrison \& Wakeman, 2000). También se han observado cambios de dominancia desde especies planctónicas a bentónicas (Philibert et al., 2003b), así como desde especies bentónicas a planctónicas (McWethy et al., 2010). Contrariamente a los efectos mencionados, Patterson et al. (2002) observaron cambios mínimos en las asociaciones de diatomeas después de incendios forestales.

\section{Incendios forestales en Patagonia Norte}

La Región de Aysén del General Carlos Ibáñez del Campo se ubica en Patagonia septentrional chilena, territorio conocido en el mundo debido principalmente a sus atractivos turísticos, donde se ha explotado su carácter de prístino, con importantes reservas de vida salvaje (Brooks et al., 2006) y gran reservorio de agua dulce (Rignot $e t$ al., 2003). Sin embargo esta zona no es totalmente prístina, ya que desde fines del siglo XIX, se realizaron exploraciones patrocinadas por el gobierno de Chile, tras las cuales se impulsó la explotación y colonización de esta zona, generándose grandes impactos ambientales (Quintanilla, 2005; Martinic, 2005). Los colonos se vieron en la necesidad de explotar el territorio, donde existía principalmente bosque nativo, muchas veces impenetrable. La principal actividad económica consistió en la introducción de ganado ovino, bovino y caballar, para lo cual fue necesario desforestar el bosque y crear praderas artificiales para el de pastoreo del ganado (Martinic, 2005). La técnica más rápida para el despeje del terreno fue la quema de bosques, cuya consecuencia inmediata resultó en el mayor periodo de incendios de origen antrópico registrado en Chile, ocurrido entre las décadas de 1930 y 1950 (Quintanilla, 2005). Según la data histórica, algunos de estos incendios duraban más de un año (Grosse, 1955; Martinic, 2005) y llegaron a consumir aproximadamente tres millones de hectáreas de bosque nativo en la Región de Aysén. Estas fueron reemplazadas en su mayoría por praderas artificiales y, posteriormente, hacia fines de la década de 1970, por plantaciones forestales (Quintanilla, 2005), evidenciándose una pérdida total aproximada del $23 \%$ de bosque nativo (Bizama et al., 2011).

No obstante lo antes señalado, existe escasa información acerca de cómo las actividades antrópicas mencionadas repercutieron sobre los ecosistemas acuáticos de la región. Dicha información es fundamental para estimar la respuesta de estos ecosistemas frente a los actuales cambios ambientales (Araneda et al., 2013), así como 
también, predecir como dichos sistemas lacustres responderían a futuro, teniendo en cuenta que, probablemente, la frecuencia e intensidad de incendios aumente como consecuencia del cambio climático global (Flannigan et al., 2001; Philibert et al., 2003a). Por lo anteriormente señalado el objetivo de este trabajo es evaluar mediante el registro sedimentario la respuesta de la comunidad diatomológica frente a los grandes incendios forestales ocurridos en Patagonia Norte durante la colonización de la zona, identificados a partir del análisis de partículas de carbón vegetal.

\section{METODOLOGÍA}

\section{Área de estudio}

El lago Thompson $\left(45^{\circ} 38^{\prime} 26^{\prime \prime} \mathrm{S} ; 71^{\circ} 47^{\prime} 07^{\prime \prime} \mathrm{W}\right)$ está ubicado aproximadamente a $20 \mathrm{~km}$ al Sureste de Coyhaique a una altitud de 750 m.s.n.m. (Fig. 1). Su cuenca tiene una superficie de $6.3 \mathrm{Km}^{2}$ y su espejo de agua cubre un área de $1.9 \mathrm{~km}^{2}$. El lago tiene una profundidad máxima de $15 \mathrm{~m}$.
La vegetación del lugar corresponde al ecotono bosque-estepa, donde predominan agrupaciones mixtas de lenga (Nothofagus pumilio) y ñirre (Nothofagus antartica) (Gajardo, 1994; Quintanilla, 2005). En la ribera oeste del lago Thompson se pueden apreciar gran cantidad de antiguos restos de árboles quemados, lo que indica que este sector fue afectado por grandes incendios, mientras que en el sector oriental del lago, predominan las plantaciones de pino (Pinus ponderosa), establecidas a partir del año 1996, que actualmente ocupan un total de 200 ha.

El lago Frío está ubicado a aproximadamente a $14 \mathrm{~km}$ al sureste de la ciudad de Coyhaique $\left(45^{\circ} 39^{\prime} 92^{\prime \prime} \mathrm{S} ; 71^{\circ} 56^{\prime} 43^{\prime \prime} \mathrm{W}\right)$ a una altitud aproximada de 530 m.s.n.m (Fig. 1). Su cuenca tiene una superficie de $124 \mathrm{~km}^{2}$ y el espejo de agua cubre un área de $4.5 \mathrm{~km}^{2}$. El lago tiene una profundidad máxima de $12 \mathrm{~m}$. La vegetación de este lugar corresponde a la misma señalada para el lago Thompson. Alrededor del lago es posible observar parches que evidencian distintos usos de suelo, incluyendo vegetación nativa, praderas y plantaciones. En su cuenca también existen plan-

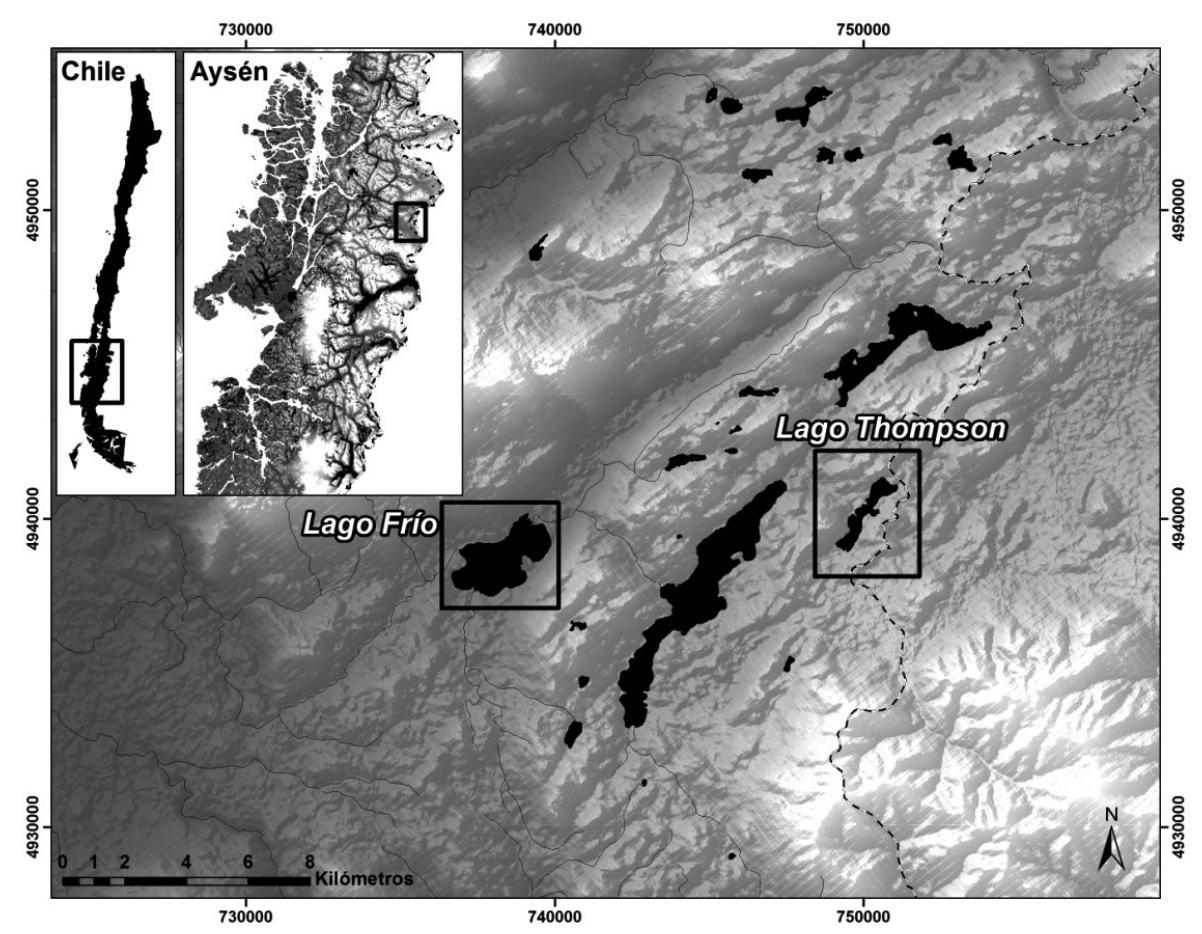

Figura 1. Área de estudio. Figure 1. Study area. 
$\mathbf{a}$

${ }^{210} \mathbf{P b}_{\text {total }}(\mathrm{pCi} / \mathrm{g})$

Edad
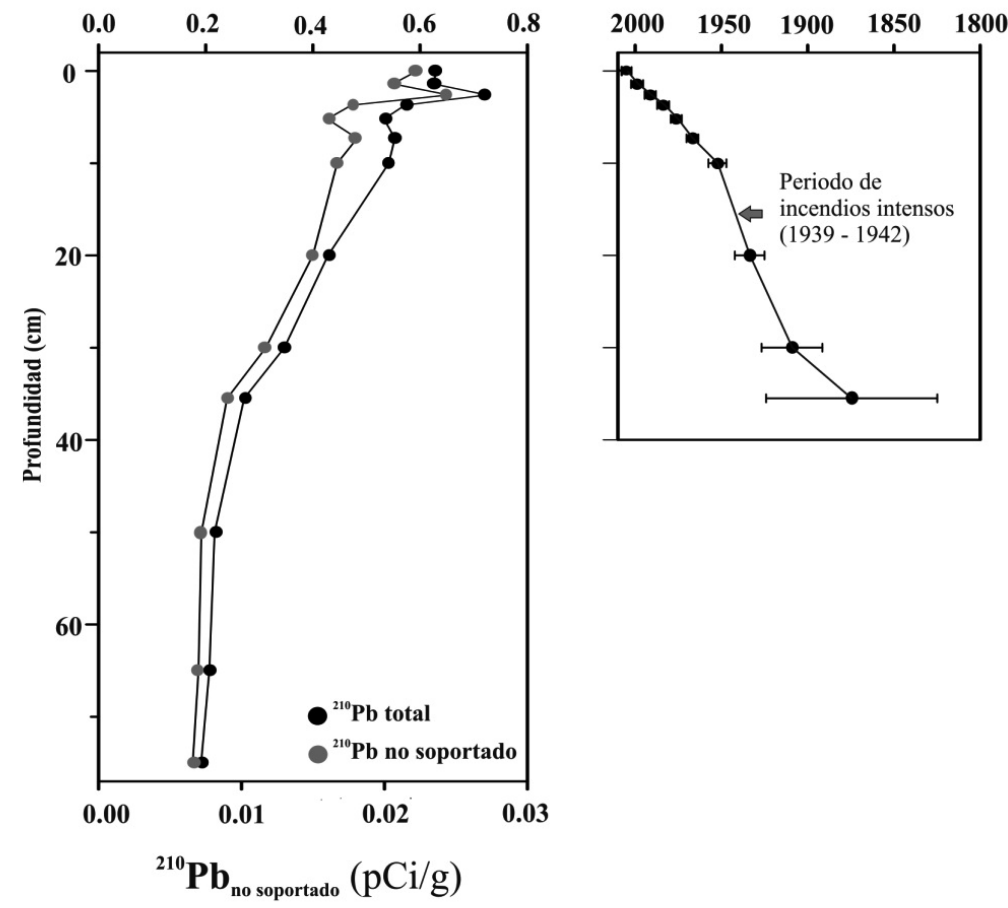

b

${ }^{210} \mathrm{~Pb}(\mathrm{mBq} / \mathrm{g})$

\section{Edad}

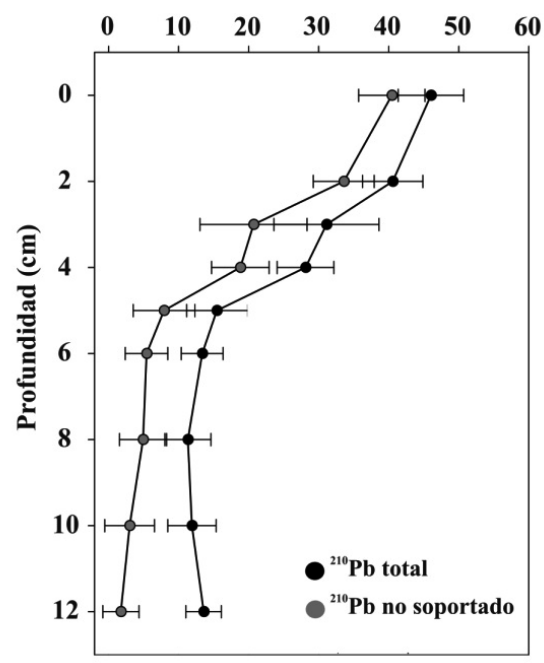

200019601920188018401800

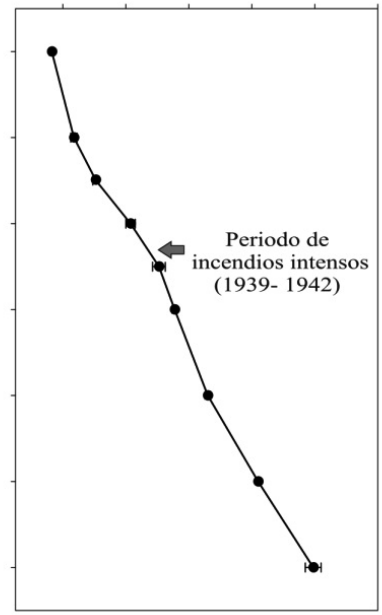

Figura 2. a) Actividad del ${ }^{210} \mathrm{~Pb}$ total y no soportado en el testigo del Lago Thompson y el modelo cronológico en el cual se indica el periodo de incendios intensos en la zona (1939-1942). b) Actividad de ${ }^{210} \mathrm{~Pb}$ total y no soportado en el testigo del lago Frío, y el modelo cronológico en el cual se indica el periodo de incendios intensos en la zona (1939-1942). a ${ }^{210} \mathrm{~Pb}$ activity (total and unsupported) in sediment core of Thompson Lake and chronologic model showing the highest fire period in the zone (1939-1942). $b)^{210} \mathrm{~Pb}$ activity (total and unsupported) in sediment core of Frio Lake, and chronologic model showing the highest fire period in the zone (1939-1942). 
taciones de Pinus ponderosa establecidas entre 1993 y 1997, ocupando un total de 255 ha, además de plantaciones de Pinus contorta.

\section{Obtención de muestras}

Los testigos de sedimento fueron colectados con un muestreador de gravedad marca Uwitec, durante el año 2008 en el lago Thompson $(40 \mathrm{~cm}$ de longitud) y 2013 en el lago Frío $(115 \mathrm{~cm}$ de longitud). En el laboratorio, los testigos fueron abiertos. El seccionamiento en el testigo del lago Thompson se realizó cada $0.5 \mathrm{~cm}$ los primeros $20 \mathrm{cms}$ y cada $1 \mathrm{~cm}$ en el resto del testigo. En el lago Frío el seccionamiento fue cada $0.5 \mathrm{~cm}$. Los análisis diatomológicos y de partículas de carbón vegetal (PCV) fueron realizados en cada sección de sedimento.

\section{Cronología}

Las edades de ambos testigos de sedimento fueron determinadas a través de la actividad de ${ }^{210} \mathrm{~Pb}$ utilizando para ello el modelo CRS (Constant Rate Supply). Este modelo asume que el aporte de ${ }^{210} \mathrm{~Pb}$ en exceso a los sedimentos es constante con el tiempo (Appleby, 2008). Para comprobar el modelo, se utilizó como trazador independiente la máxima concentración de partículas de carbón registrada en cada núcleo de sedimento. En Patagonia norte, los eventos de incendios han sido bien definidos a través del registro histórico, estableciéndose que entre 1939 y 1942 ocurrieron los más intensos.

\section{Análisis de partículas de carbón (PCV)}

Para evidenciar periodos de incendios a lo largo del testigo sedimentario se realizaron análisis de PCV en el lago Frío, mientras que los resultados de PCV del lago Thompson fueron obtenidos de Araneda et al. (2013). En cada estrato se preparó la muestra según la metodología propuesta por Lynch et al. (2003). Luego la muestra se lavó en un tamiz de $125 \mu \mathrm{m}$ (Whitlock, 2001) para ser analizada en una lupa con $36 \times$ de aumento. La concentración de PCV se estimó dividiendo el número de partículas contadas por el volumen de sedimento utilizado. Luego se determinó la tasa de acumulación de PCV, multiplicando la concentración en unidad de área por la tasa de sedimentación (Whitlock \& Millspaugh, 1996).

\section{Análisis Diatomológicos}

Para ambos lagos, se efectuó la oxidación de la materia orgánica y eliminación de las sales disueltas, utilizando la metodología propuesta por Battarbee (1986). Posteriormente se realizaron muestras permanentes utilizando resina Naphrax $(\mathrm{I} . \mathrm{R}=1.7)$ como medio de montaje. En cada estrato de la columna de sedimento se contaron e identificaron aproximadamente 500 valvas de diatomeas, utilizando un microscopio Carl Zeiss Axioplan, con objetivo de inmersión de $100 \times$. Además se utilizó un microscopio electrónico de barrido JEOL/DEO para una revisión más detallada de las especies. La taxonomía y revisión autoecológica de las especies se basó en literatura especializada para diatomeas (Barber \& Carter, 1996; Krammer \& Lange-Bertalot, 2000; Rumrich et al., 2000). También se obtuvo la abundancia absoluta en cada estrato siguiendo la metodología de Battarbee (1986); se estimaron las abundancias relativas de cada especie por estrato, la riqueza, diversidad (índice de Shannon-Wienner $H^{\prime}$ ), dominancia (Simpson), y razón diatomeas planctónicas/bentónicas $(\mathrm{P} / \mathrm{B})$.

\section{Análisis de datos}

Para distinguir las diferentes asociaciones a lo largo del perfil, se aplicó un análisis de clúster limitado estratigráficamente (CONISS) a los valores de abundancias relativas de las asociaciones diatomológicas, utilizando los programas TILIA y TILIA GRAPH (Grimm, 1987).

Luego se realizaron perfiles estratigráficos de parámetros comunitarios de diatomeas (abundancia, diversidad y dominancia), razón P/B y PCV utilizando el Programa Sigmaplot 12. Se realizó además un análisis DCA (detrended correspondence analysis) a la información diatomológica para evaluar cambios en las asociaciones usando el programa R. Posteriormente se efectuó un análisis de escalamiento multidimensional 

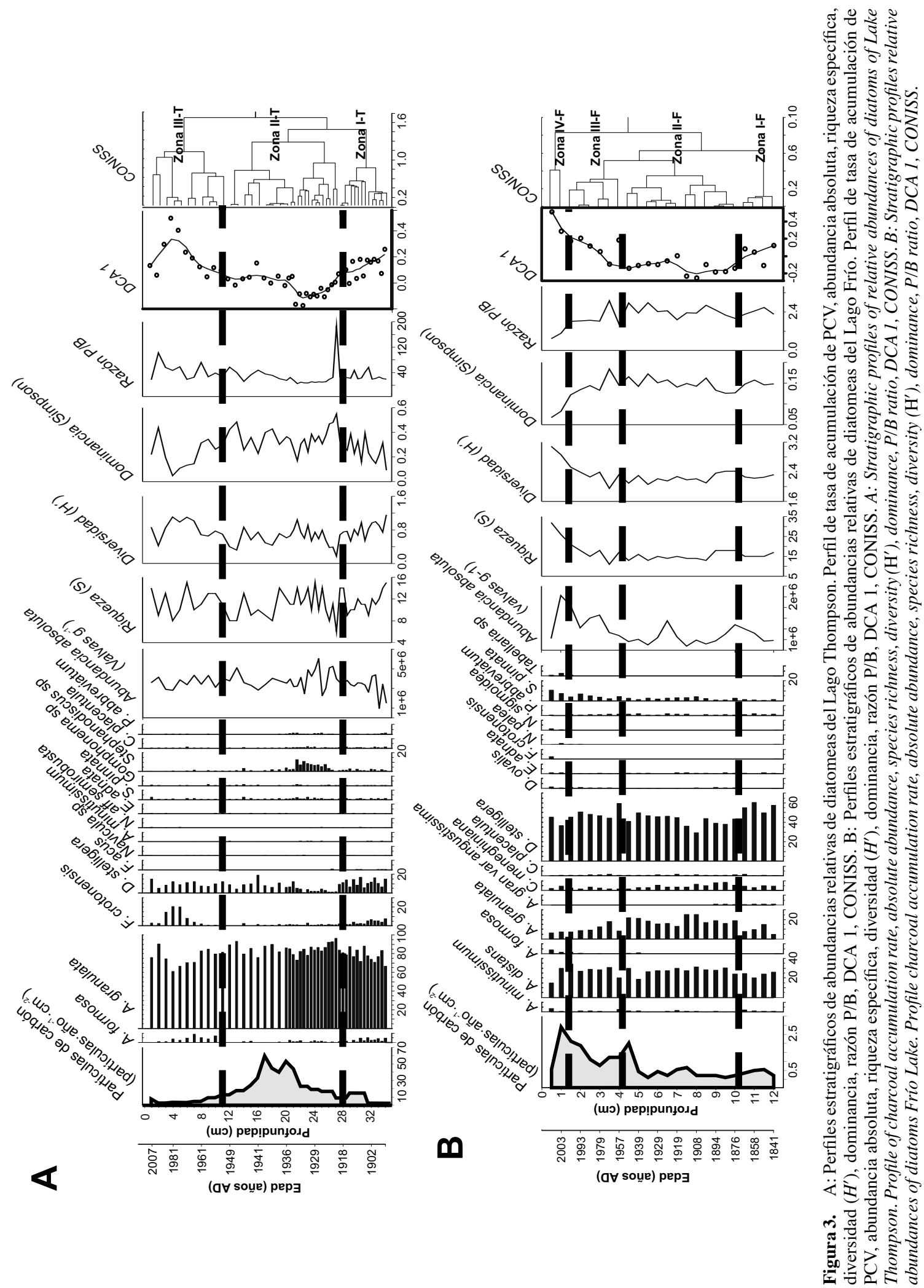
(MDS, por sus siglas en inglés) para evidenciar cambios entre periodos de pre-incendios, incendios y post-incendios. La significancia de cambios entre los periodos se determinó mediante un análisis de similitud (ANOSIM, Clarke \& Warwick, 2001), utilizando el Programa PRIMER. Finalmente se efectuó un análisis no paramétrico Kruskal-Wallis para evidenciar diferencias estadísticas de los parámetros comunitarios y razón $\mathrm{P} / \mathrm{B}$ entre periodos, utilizando el programa Statistica.

\section{RESULTADOS}

\section{Cronología}

La actividad del ${ }^{210} \mathrm{~Pb}$ total y el no soportado en ambos núcleos sedimentarios presenta un decaimiento radioactivo relativamente constante con la profundidad. La utilización del modelo CRS permitió estimar la edad de los sedimentos para ambos núcleos. Para el lago Thompson, el núcleo obtenido permitió registrar cambios de los últimos 135 años, en tanto que en el lago Frío el núcleo sedimentario permitió obtener un registro desde 1840. Las edades determinadas a través del modelo cronológico coincidieron con la máxima concentración de PCV, siendo coherentes con los periodos de incendios intensos documentados en la zona (Fig. 2).

\section{Partículas de carbón vegetal}

Las PCV en ambos lagos fueron observadas a lo largo de todo el perfil sedimentario, sin embargo sus concentraciones muestran diferencias importantes. En el lago Thompson la concentración de PCV (Fig. 3A) fluctuó entre 3 a 60 partículas $\cdot a_{n} o^{-1} \cdot \mathrm{cm}^{-2}$, encontrándose una mayor concentración entre 1923 (cm 26.5) y 1945 (cm 14), con dos máximos alrededor de 1936 (cm 20) con 55 partículas $\cdot$ año ${ }^{-1} \cdot \mathrm{cm}^{-2}$ y en 1940 (cm 17) con 60 partículas $\cdot$ año ${ }^{-1} \cdot \mathrm{cm}^{-2}$. Posteriormente, la tasa de acumulación de PCV tiende a disminuir hasta 1961, donde se estabiliza el flujo hasta el periodo más reciente del testigo con aproximadamente 3 partículas $\cdot \mathrm{año}^{-1} \cdot \mathrm{cm}^{-2}$.
En el lago Frío (Fig. 3B) la tasa de acumulación de PCV fue más baja, con valores que fluctuaron

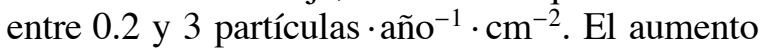
más importante de PCV es observado posterior a 1939 (cm 5), donde se evidencia una mayor concentración en 1946 con 2 partículas $\cdot$ año ${ }^{-1} \cdot \mathrm{cm}^{-2}$ (cm 4.5) y en 2003 ( $\mathrm{cm} \mathrm{1),} \mathrm{donde} \mathrm{se} \mathrm{encontraron}$ 3 partículas $\cdot \mathrm{año}^{-1} \cdot \mathrm{cm}^{-2}$.

\section{Diatomeas}

\section{Lago Thompson}

Se contaron e identificaron un total aproximado de 24000 valvas de diatomeas (500/muestra) en 48 muestras. Se identificaron en total 40 taxones. Las especies más abundantes a lo largo del perfil fueron Asterionella formosa, Aulacoseira granulata, Fragilaria crotonensis, Discostella stelligera y Stephanodiscus sp.

A través del análisis CONISS fue posible distinguir 3 zonas para los últimos 118 años (Fig. 3A), las que se presentan a continuación como:

- Zona I-T periodo 1889-1917 (34-27.5). La zona I se caracteriza principalmente por la presencia de $A$. formosa, $F$ crotonensis, $D$. stelligera y $A$. granulata siendo esta última la más abundante en la zona. Por otro lado, se observa que la abundancia total absoluta es baja, y alcanza el menor valor $\left(7.5 \cdot 10^{5}\right.$ valvas/g) en 1896 ( $\mathrm{cm} \mathrm{33).} \mathrm{La} \mathrm{riqueza} \mathrm{y}$ diversidad presentaron valores promedios de 11 y 0.9 , respectivamente, mientras que los valores de dominancia fueron cercanos a 0.3 , y una razón $\mathrm{P} / \mathrm{B}$ de 0.4 , la cual no varía considerablemente.

- Zona II-T periodo 1918-1952 (cm 28-11). En la zona II se observa una disminución de la abundancia de A. formosa, F. crotonensis y D. stelligera, estando ausentes en algunos estratos del periodo. Por el contrario la abundancia de $A$. granulata se mantiene relativamente estable, mostrando un leve aumento en 1922 (cm 27), donde alcanza el $96 \%$ (cm 27). Posteriormente, la leve disminución de A. granulata, coincide con el aumento de Stephanodiscus sp. en 1934 (cm 21.5) con 
un $12 \%$ y con el aumento de la abundancia absoluta con un pico de $4.8 \cdot 10^{6}$ valvas/g en 1928 (cm 24.5). Los índices de riqueza y diversidad muestran un valor promedio de $10 \mathrm{y}$ 0.8 , los cuales son levemente más bajos que en la zona I. La dominancia y la razón P/B, aumentan drásticamente alrededor de 1920, pero tienden a disminuir rápidamente y mantenerse con valores más bajos al final de esta.

- Zona III-T periodo 1954-2007 (cm 10-1). En esta zona se observa un aumento de la abundancia de A. formosa, F. crotonensis y D. stelligera y una disminución de A. granulata y Stephanodiscus sp. en comparación a la zona II. La abundancia total se mantiene relativamente constante, mientras que los índices de riqueza y diversidad tienden a aumentar levemente posterior a 1961. Contrariamente, la dominancia, posterior a este año, disminuye y la razón $\mathrm{P} / \mathrm{B}$ tiende a aumentar al final de la zona.

\section{Lago Frío}

Se contaron e identificaron al mínimo nivel taxonómico posible (género/especie) un total aproximado de 20000 valvas de diatomeas (500/muestra) en 40 muestras. Se encontraron en total 58 taxones. Las especies más abundantes a lo largo del perfil fueron Aulacoseira distans, Aulacoseira granulata, Aulacoseira granulata. var angustissima, Cyclotella meneghiniana, Discostella stelligera y Staurosirella pinnata.

A través del análisis CONISS fue posible distinguir 4 zonas para los últimos 170 años (Fig.3B), las que se presentan a continuación como:

- Zona I-F periodo 1841-1867 (cm 12-10.5). Esta zona se caracteriza por la presencia de A. distans, A. granulata, C. meneghiniana y D. stelligera, siendo esta última la que se encuentra en mayor abundancia con casi un $60 \%$. La abundancia absoluta tiende a aumentar levemente hacia el final del periodo y los índices de riqueza y diversidad se mantienen estables con valores promedio de 15 y 2.2 , respectivamente. Solo la dominancia y la razón $\mathrm{P} / \mathrm{B}$ evidenciaron un leve aumento en la mitad del periodo y posteriormente tendieron a disminuir.

- Zona II-F periodo 1876-1948 (cm 10-4.5). Los taxones característicos de esta zona son los mismos descritos para la zona I. Las abundancias de $A$. distans, $A$ granulata y $D$. stelligera se mantienen relativamente constantes, solo $C$. meneghiniana muestra una disminución de su abundancia hacia el final del periodo. De los parámetros comunitarios solo los valores de abundancia absoluta y razón $\mathrm{P} / \mathrm{B}$ muestran variaciones a lo largo de la zona. Una disminución de la abundancia absoluta se evidencia entre 1894 y 1919 (cm 9 y 7), coincidiendo con el leve aumento de la razón P/B y la abundancia de $A$. granulata entre 1908 y 1919, así como con la disminución de D. stelligera. Posterior a esta fecha, la abundancia absoluta aumenta hasta 1929, donde vuelve a disminuir y se mantiene baja hasta el final del periodo. Contrariamente, la razón P/B después de 1929 comienza a incrementar.

- Zona III-F periodo 1957-1998 (cm 4-1.5). En esta zona, A. distans y D. stelligera son las especies dominantes, con valores de abundancia de 30 y $50 \%$ respectivamente, las cuales se mantienen relativamente constantes durante este periodo. Contrariamente, A. granulata muestra una tendencia a disminuir su abundancia, mientras que $S$. pinnata incrementa. La abundancia absoluta evidencia un continuo aumento hacia la parte superior de la zona, observándose solo una leve disminución entre 1979 y 1993. Los índices de riqueza y diversidad tienden a incrementar, pero evidencian una disminución en sus valores en un periodo anterior (1957-1979) al de la disminución de abundancia, lo cual coincide con un aumento de la dominancia y la razón $\mathrm{P} / \mathrm{B}$, los que posteriormente tienden a disminuir hacia el final de la zona.

- Zona IV-F periodo 2003-2008 (cm 1-0.5). Durante este periodo, se observa un aumento 
importante en la abundancia absoluta. Las especies dominantes continúan siendo $A$. distans y D. stelligera. Sin embargo, esta zona se caracteriza por la aparición de nuevas especies, como F. crotonensis, Nitzschia palea, Nitzschia sigmoidea y Tabellaria sp., que se encuentran en bajos porcentajes. Los parámetros comunitarios, como la riqueza $\mathrm{y}$ diversidad alcanzan los mayores valores, mientras que la dominancia y la razón $\mathrm{P} / \mathrm{B}$ muestran los valores más bajos de todo el perfil.

\section{Diferencias estadísticas entre periodos}

Los periodos de pre-incendios, incendios y postincendios, fueron determinados a partir de las mayores concentraciones de PCV en los perfiles sedimentarios de ambos lagos. Para el lago Thompson, el periodo de incendios corresponde a la zona II-T (1918-1952), el de pre-incendios a la zona I-T y de post-incendios a la zona III-T. Coincidentemente, el DCA evidencia cambios en la zona II-T, con valores menores a cero, mientras que en los otros periodos los valores
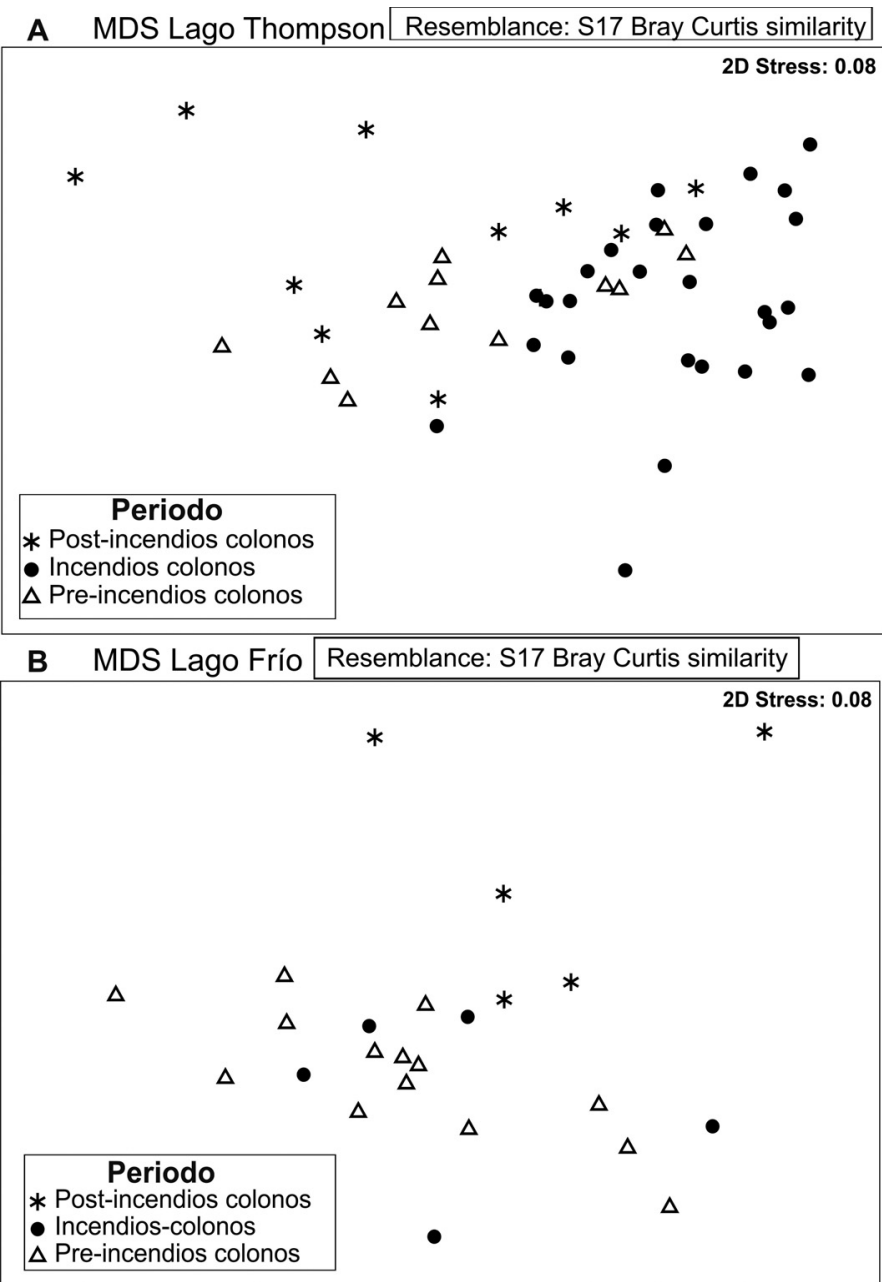

Figura 4. Análisis MDS de diatomeas registradas en los lagos Thompson y Frío. Los círculos abiertos representan periodos de posibles incendios previos a los ocasionados por los colonos, los triángulos abiertos representan periodos de pre-incendios de colonos, los círculos cerrados representan incendios durante la colonización, y los asteriscos representan el periodo de post- incendios de los colonos. MDS Analysis of diatoms recorded in the Thompson and Frío lakes. The open circles represent periods of possible previous fires caused by the settlers, open triangles represent periods of pre-settler fires, closed circles represent fire during colonization, and the asterisks represent the period of post-fire settlers. 
son cercanos a 0.2. El agrupamiento en el MDS (Fig. 4A), muestra similitudes entre periodos de pre y post incendios y una menor similitud con el periodo de incendios. Aunque el test ANOSIM, entregó diferencias significativas entre los periodos $\left(R_{\text {global }}=0.33, p=0.001\right)$, el test pairwise mostró que las diferencias sólo ocurrieron entre el periodo de incendios y preincendios $(R=0.28, p=0.03)$ y el periodo de incendios y post-incendios $(R=0.46, p=0.01)$, no encontrándose diferencias significativas entre periodos de pre y post incendios $(R=0.085$, $p=0.1$ ). Las diferencias estadísticas de los parámetros comunitarios y razón $\mathrm{P} / \mathrm{B}$ en los distintos periodos mostraron diferencias significativas sólo en la razón $\mathrm{P} / \mathrm{B}$, las que se dieron entre el periodo de incendios y pre-incendios y el periodo de incendios y post-incendios (Tabla 1).

En el lago Frío (Fig 3B), el periodo de eventos incendiarios resultante de la colonización de la zona, fue identificado entre 1939 y 1979 (cm 5$3)$, estableciéndose un periodo pre-incendios entre 1941-1931 (cm 12-5.5) y post-incendios entre 1985 y 2012 (cm 2.5-0). Coincidentemente, el cambio más importante en la asociación de dia- tomeas, evidenciado a través del DCA, ocurre durante el periodo de incendios con valores negativos (Fig. 3B). El ordenamiento de los periodos en el MDS (Fig. 4B) muestra una mayor similitud entre el periodo de pre-incendios e incendios y una mayor disimilitud de ambos periodos con el periodo de post-incendios. El test ANOSIM mostró diferencias estadísticamente significativas $\left(R_{\text {global }}=0.26, p=0.02\right)$, mientras que el test pairwise indicó que las diferencias significativas ocurrieron entre el periodo de incendios y post-incendios $(R=0.23, p=0.02)$ y entre pre-incendios y post-incendios $(R=0.45$, $p=0.005)$. Las diferencias estadísticas de los parámetros comunitarios y razón $\mathrm{P} / \mathrm{B}$ en los distintos periodos fueron observadas en el índice de riqueza y razón $\mathrm{P} / \mathrm{B}$ entre el periodo de preincendios y post-incendios (Tabla 1).

\section{DISCUSIÓN}

Los registros sedimentarios de los dos lagos estudiados permitieron evidenciar cambios en las asociaciones diatomológicas durante los últimos

Tabla 1. Significancia estadística de cambios en parámetros comunitarios de abundancia absoluta, riqueza, diversidad, dominancia y razón P/B en los distintos periodos, para los lagos Thompson y Frío, basados en el test estadístico no paramétrico Kruskal-Wallis. Statistical significance of changes in community parameters such as absolute abundance, richness, diversity, dominance and P/B ratio in different periods, for Thompson and Frio lakes, based on non-parametric Kruskal-Wallis test.

\begin{tabular}{|c|c|c|c|c|}
\hline \multicolumn{5}{|c|}{ Lago Thompson } \\
\hline Parámetro & & $\begin{array}{l}\text { ndios- } \\
\text { cendios }\end{array}$ & $\begin{array}{l}\text { Incendios- } \\
\text { post-incendios }\end{array}$ & $\begin{array}{l}\text { Pre-incendios- } \\
\text { post-incendios }\end{array}$ \\
\hline Abundancia absoluta & & 0.23 & $p=1$ & $p=1$ \\
\hline Riqueza & & 0.77 & $p=0.67$ & $p=1$ \\
\hline Diversidad & & $=1$ & $p=0.71$ & $p=0.86$ \\
\hline Dominancia & & $=1$ & $p=1$ & $p=1$ \\
\hline Razón P/B & & 0.01 & $p=0.00$ & $p=0.55$ \\
\hline \multicolumn{5}{|c|}{ Lago Frío } \\
\hline Parámetro & $\begin{array}{l}\text { Incendios colonos- } \\
\text { pre-incendios colonos }\end{array}$ & $\begin{array}{c}\text { Incendios colonos- } \\
\text { post-incendios colonos }\end{array}$ & $\begin{array}{l}\text { Pre-incendios colonos- } \\
\text { post-incendios colonos }\end{array}$ & $\begin{array}{c}\text { Incendios pre-colonización- } \\
\text { Pre-incendios colonos }\end{array}$ \\
\hline Abundancia absoluta & $p=1$ & $p=0.73$ & $p=0.07$ & $p=0.00$ \\
\hline Riqueza & $p=1$ & $p=0.27$ & $p=0.02$ & $p=1$ \\
\hline Diversidad & $p=1$ & $p=0.16$ & $p=0.39$ & $p=0.03$ \\
\hline Dominancia & $p=1$ & $p=1$ & $p=1$ & $p=0.23$ \\
\hline Razón P/B & $p=1$ & $p=0.50$ & $p=0.00$ & $p=1$ \\
\hline
\end{tabular}


170 años. Sin embargo su relación con los eventos incendiarios generados por lo colonos fue diferente para ambos lagos. Estas diferencias podrían estar asociadas a los distintos flujos de PCV registrados en los sedimentos, que fueron significativamente mayores en el lago Thompson. Las pendientes de las cuencas podrían ser uno de los factores con mayor influencia en la deposición de PCV ya que aunque morfológicamente el lago Frío es de mayor tamaño que el lago Thompson, la mayor pendiente en este último, pudo haber sido favorable para una mayor escorrentía y con ello una mayor deposición de PCV en el sedimento. Esta situación además, sería coincidente con la mayor tasa de sedimentación que presentó el lago Thompson.

Las relaciones encontradas en el lago Thompson entre los periodos de mayor flujo de PCV y las asociaciones de diatomeas permitieron establecer que el periodo de incendios correspondió a la zona II-T identificada a través de las asociaciones diatomológicas (Fig. 3A). Los cambios en las asociaciones de diatomeas en este lago, reflejadas en su composición especifica fueron similares a lo reportado en otros estudios (Enache \& Prairie, 2000; Philibert et al., 2003b; McWethy, 2010). En el lago Thompson, estos cambios se reflejaron principalmente en el aumento de la abundancia de Stephanodiscus sp.,antes de la máxima acumulación de PCV así como en una disminución de A. formosa, F. crotonensis y D. stelligera, lo cual puede ser asociado a un cambio a condiciones altamente eutróficas (Lei et al., 2008; Makarewicz \& Baybutt, 1981), considerando además que estas tres especies han sido reportadas en lagos oligotróficos alpinos (Saros et al., 2005).

La disminución de Stephanodiscus sp. y $F$. crotonensis después del periodo con mayor eventos incendiarios evidenciados por el pico de PCV coincide con lo reportado por Philibert et al., (2003a, 2003b), quienes indican que la disminución de ambas especies ocurre como una respuesta a los incendios. Según Enache \& Prairie (2000), los cambios observados en las asociaciones de diatomeas podrían estar relacionados con el aumento de nutrientes, principalmente de fósforo $(\mathrm{P})$, por lo que es posible que durante y posterior al incendio, el lago Thompson presentara una mayor concentración de estos, favoreciendo la abundancia de especies que habían disminuido en el periodo anterior.

Pese a que hubo diferencias significativas en cuanto a la composición de especies entre el periodo de incendios y los periodos de pre y postincendios, los parámetros comunitarios tales como abundancia absoluta, riqueza, diversidad, dominancia, y la razón P/B no mostraron la misma tendencia. La riqueza específica no mostró un cambio significativo, coincidiendo con lo reportado por Philibert et al. (2003b) y contrario a los estudios de Enache \& Prairie (2000) y Rosén \& Hammarlund (2007) quienes evidenciaron una importante disminución de la riqueza en periodos de incendios. La abundancia absoluta tampoco mostró cambios significativos, lo que no coincide con el trabajo de Philibert et al. (2003b) quienes señalan un aumento de la abundancia luego de los incendios.

La ausencia de cambios significativos en los índices de diversidad y dominancia como respuesta al periodo de incendios fue contrario a lo esperado, debido a que Watchorn et al. (2008) registran cambios en estos parámetros como resultado de la pérdida de vegetación y subsecuente aumento de nutrientes. Finalmente el único parámetro que presentó diferencias significativas entre periodos fue la razón $\mathrm{P} / \mathrm{B}$, observándose un aumento durante el periodo de incendios. Este cambio coincide con lo observado por McWethy et al. (2010), quienes plantean que el incremento de material terrestre al lago, derivado de los incendios, puede reducir la disponibilidad de luz en el fondo del mismo y con ello reducir el área de hábitats bentónicos. Sin embargo en este estudio se debe tener presente que la razón $\mathrm{P} / \mathrm{B}$ aumento drásticamente sólo al comienzo del periodo de incendios, y el aumento no fue una tendencia general del periodo. Posiblemente, los primeros incendios en la zona, aunque pudieron haber sido de menor intensidad, generaron una entrada importante de material al lago, lo que se vio reflejado en una disminución de especies bentónicas y aumento de especies planctónicas, principalmente de A. granulata (Fig. 3A).

En cuanto al tiempo de recuperación de la asociación de diatomeas se puede apreciar que las 
especies que cambiaron su abundancia durante el periodo de incendios, volvieron a tener abundancias similares al periodo de pre-incendios en un lapso de entre 5 (A. formosa) a 10 años (F. crotonensis) tal como se ha reportado en varios estudios (Bayley et al., 1992; Philibert et al., 2003b; Temporetti, 2006). Esto sugiere que las respuestas limnológicas frente a eventos de incendios serían de corta duración, lo que permitiría a las comunidades de diatomeas recuperar su estructura una vez que los efectos de los incendios desaparecen. Por otra parte, también es posible señalar que hubo especies como $D$. stelligera, que presentan una mayor adaptación a los cambios en el lago, por lo que recuperan su abundancia con mayor rapidez.

Contrario al lago Thompson, la respuesta diatomológica en el lago Frío no fue claramente evidenciada a través de las diferencias encontradas entre las zonas que entregó el análisis de clúster, basados en las asociaciones de diatomeas y los periodos de pre-incendios, incendios y postincendios. Además, las diferencias significativas de las asociaciones solo fueron encontradas entre el periodo de incendios generado por los colonos y el de post-incendios, y entre un periodo de incendios ocurrido previo a la colonización, con periodos posteriores. De la misma forma, la respuesta de los parámetros comunitarios no fue clara, presentándose cambios significativos en la riqueza y razón $\mathrm{P} / \mathrm{B}$ entre el periodo de pre-incendios y post-incendios, así como cambios significativos en la abundancia absoluta y diversidad entre el periodo de incendios previos a la colonización y el periodo de pre-incendios de los colonos. Teniendo en cuenta lo anterior, se podría señalar que los cambios observados estarían respondiendo a la dinámica propia del cuerpo de agua, influida probablemente por otros factores entre los cuales podrían encontrarse variaciones climáticas (Szeicz et al., 1998).

Los cambios registrados durante el periodo de incendios previos a la colonización (ca. 1867-1900), y que fueron evidenciados por el mayor cambio del DCA y un posterior aumento de la abundancia de A. granulata var. angustissima, probablemente tengan relación con un periodo frío previamente documentado para esta zona (Araneda et al., 2007), debido a que dichos cambios ocurrieron durante este evento climático y duraron aproximadamente hasta el final del periodo frío (ca. 1904). Similarmente, Szeicz et al. (1998) estudiando la laguna Venus, localizada en una zona cercana al área de este estudio, sugieren que las variaciones climáticas podrían haber influido en las asociaciones de crisófitas de este cuerpo de agua, cuyos cambios no coincidían con los mayores flujos de PCV registrados en el testigo de sedimento.

En general los resultados del lago Frío parecen no ser tan diferentes de otras investigaciones, en las cuales tampoco se encontró un impacto sustancial en las comunidades de diatomeas como respuesta a los incendios (Paterson et al., 1998; 2002; Moser et al., 2002; Philibert et al., 2003a). Una posible explicación podría ser la baja tasa de sedimentación (Philibert et al., 2003a), que en el lago Frío fue de aproximadamente 16 años por centímetro. A esta situación también se podría atribuir la baja concentración de PCV, comparativamente con lo encontrado en el lago Thompson. Además, considerando que los incendios podrían generar cambios en las características del lago durante un periodo relativamente corto de tiempo, los efectos sobre las asociaciones de diatomeas podrían no ser detectados, debido a que la información de tales perturbaciones podría estar registrada solo en unos pocos estratos del sedimento. Además, Schindler (1980), señalan que posterior a un incendio, los cambios en la química del agua son de corta duración, lo que provoca que no se presenten efectos significativos en las concentraciones del fitoplancton.

La susceptibilidad de los sistemas acuáticos a perturbaciones como los eventos de incendios, pareciera depender en parte del estado trófico del lago y también a factores geomorfológicos, como el volumen y batimetría del lago y pendiente de la cuenca (Enache \& Prairie, 2000). En este caso, las condiciones tróficas del lago Thompson pudieron ser el factor determinante. Antes de los incendios, la presencia de A. formosa y $F$. crotonensis estarían sugiriendo la prevalencia de condiciones mesotróficas en este lago, los cuales producto de la ocurrencia de los incendios pudieron haber alcanzado rápidamente niveles de una 
condición eutrófica, que además pudo haber sido favorecida con el cambio de uso de suelo ocurrido en la actualidad (plantaciones forestales).

Lo anterior se ve reforzado por Araneda et al. (2013) quienes en el mismo lago infirieron a través de las asociaciones de quironómidos que este estado trófico comenzó con los eventos de incendios asociados a la colonización de Patagonia Norte y mantuvo esta condición hasta la actualidad. Por otro lado, los cambios climáticos también podrían haber influido en los cambios de las asociaciones diatomológicas, no obstante, esto es difícil de establecer de forma directa debido a que se esperaría que las variaciones climáticas fueran registradas sincrónicamente en ambos lagos.

\section{CONCLUSIONES}

El Lago Thompson presentó una mayor sensibilidad a los efectos derivados de los incendios en la composición de las asociaciones de diatomeas, en comparación al lago Frío, verificándose un recambio de especies, excepto en Stephanodiscus sp. y Discostella stelligera, que reaparecen antes que finalizara el periodo de incendios. Esto pudo deberse a un mayor aporte de fósforo inmediatamente luego de ocurrido los incendios o también a que éstas especies se adaptaron más rápidamente a los cambios en la calidad del agua.

Las disimilitudes entre los lagos, se debieron probablemente a diferencias en la resolución temporal, a un diferente estado trófico antes de los incendios y a factores como el volumen del lago y pendiente de la cuenca. La baja resolución temporal del lago Frío, podría no haber permitido registrar claramente los cambios en las diatomeas, debido a que cambian rápidamente tanto frente a cambios ambientales, como al retorno a condiciones previas. Por otra parte, el lago Thompson habría presentado un estado trófico más alto que el lago Frío, antes de la ocurrencia de los incendios, lo que sumado a su menor volumen y mayor pendiente de su cuenca, pudieron haberle llevado a una condición eutrófica, rápidamente luego de los incendios.

Finalmente, se concluye también que los parámetros comunitarios estudiados no son bue- nos indicadores del cambio en estos ecosistemas, frente a perturbaciones causadas por incendios forestales, al menos en este tipo de investigaciones.

\section{AGRADECIMIENTOS}

FONDECYT N ${ }^{\circ} 1120765, \mathrm{~N}^{\circ} 1120807$ y a Alex Henríquez por su contribución en la generación del área de estudio.

\section{REFERENCIAS}

ALEFS, J. \& J. MÜLLER. 1999. Differences in the eutrophication dynamics of Ammersee and Starnberger See (southern Germany), reflected by the diatom succession in varve-dated sediments. Journal of Paleolimnology, 21: 395-407.

ANDERSON, N., E. JEPPESEN \& M. SONDERGAARD. 2005. Ecological effects of reduced nutrient loading (oligotrophication) on lakes: an introduction. Freshwater Biology, 50: 1589-193.

APPLEBY, P. 2008. Three decades of dating recent sediments by fallout radionuclides: a review. $\mathrm{Ho}$ locene, 18: 83-93.

ARANEDA, A., F. TORREJÓN, M. AGUAYO, L. TORRES, F. CRUCES, M. CISTERNAS \& R. URRUTIA. 2007. Historical record of San Rafael glacier advances (North Patagonian Icefield): another clue for Little Ice Age timing in Souther Chile. The Holocene, 17: 987-998.

ARANEDA, A., P. JANA, C. ORTEGA, F. TORREJÓN, S. BERTRAND, P. VARGAS, F. FAGEL, D. ALVAREZ, A. STEHR \& URRUTIA R. 2013. Changes in sub-fossil chironomid assemblages in two Northern Patagonian lake systems associated with the occurrence of historical fires. Journal of Paleolimnology, 50(1): 41- 56.

BARBER, H. \& J. CARTER. 1996. An atlas of British diatoms. Biopress Limited, Bristol, UK.

BATTARBEE, R. 1986. Diatom analysis. In: Handbook of Holocene Paleoecology and Paleohidrology. E. BB (ed). 527-570. John Wiley \& Sons Ltd. Chichester. UK.,.

BAYLEY, S., D.W. SCHINDLER, K. BEATY, B. PARKER \& M. STAINTON. 1992. Effects of multiple fires on nutrient yields from streams draining boreal forest and fen watersheds: nitrogen 
and phosphorus. Canadian Journal of Fisheries Aquatic Science, 49: 584-596.

BERGERON, Y., A. LEDUC, B. HARVEY \& S. GAUTHIER. 2000. Natural fire regime: A guide for sustainable forest management of the boreal forest. SFM Network 2000-13, Edmonton, Alberta, Canadá.

BIZAMA, G., F. TORREJÓN, M. AGUAYO, M. MUÑOZ, E. ECHEVERRÍA \& R. URRUTIA R. 2011. Pérdida y fragmentación del bosque nativo en la cuenca del río Aysén (Patagonia-Chile) durante el siglo XX. Revista de Geografía Norte Grande, 49: 125-138.

BROOKS, T., R. MITTERMEIER, G. FONSECA, J. GERLACH, M. HOFFMANN, J. LAMOREUX, C. MITTERMEIER, J. PILGRIM \& A. RODRIGUES. 2006. Global biodiversity conservation priorities science. Science, 313: 58-61.

CLARKE, K. \& R. WARWICK. 2001. Change in marine communities: an approach to statistical analysis and interpretation. $1^{\text {st }}$ edn. Plymouth Marine Laboratory, Plymouth, UK; $2^{\text {nd }}$ edn. PRIMER-E, Plymouth, UK

ENACHE, M. \& Y. PRAIRIE. 2000. Paleolimnological reconstruction of forest fire induced changes in lake biogeochemistry (Lac Francis, Abitibi, Quebec, Canada). Canadian Journal of Fisheries Aquatic Science, 57(2): 146-154.

FLANNIGAN, M., I. CAMPBELL, B. WOTTON, C. CARCAILLET, P. RICHARD \& Y. BERGERON. 2001. Future fire in Canada's boreal forest: palaeoecology results and general circulation model - regional circulation model simulations. Canadian Journal Forest Research, 31: 854-864.

GAJARDO, R. 1994. La vegetación natural de Chile clasificación y distribución geográfica. Editorial Universitaria. Santiago, Chile.

GARRISON, P. \& S. WAKEMAN. 2000. Use of paleolimnology to document the effect of lake shoreland development on water quality. Journal of $\mathrm{Pa}$ leolimnology, 24: 369-393.

GRIMM, E. 1987. A Fortran 77 program for stratigraphically constrained cluster analysis by the method of incremental sum of squares. Computer and Geosciences, 13: 13-35.

GROSSE, A. 1955. Visión de Aysén. Expediciones del Explorador Juan Augusto Grosse. Ed. del autor. Santiago, Chile.

KRAMMER, K. \& H. LANGE-BERTALOT. 2000. Siisswasserflora von Mitteleuropa Bacillariophy- ceae Teil: Centrales, Fragilariaceae, Eunotiaceae. Fischer Verlag, Stuttgart, Stuttgart, Germany.

LANGMAN, R. 1971. Patterns of settlement in Southern Ontario. McClelland and Stewart Limited. Toronto, Canadá.

LYNCH, J., J. CLARK, N. BIGELOW, M. EDWARDS \& B. FINNEY. 2003. Geographic and temporal variations in fire history in boreal ecosystems of Alaska. Journal of Geophysical Research, 108(8152): 8.1-8.17.

LEI, L., L. LA-MEI, X. LI-JUAN \& H. BO-PING. 2008. Dynamics of the trophic state and phytoplankton community of a small reservoir in South China. Ecological Science, 27: 71-76.

MAKAREWICZ, J. \& R. BAYBUTT. 1981. Longterm (1927-1978) changes in.the phytoplankton community of Lake Michigan at Chicago. Bulletin of the Torrey Botanical Club, 108: 240-254.

MARKGRAF, V., C. WHITLOCK \& S. HABERLE. 2007. Vegetation and fire history during the last 18,000 cal yr B.P. in Southern Patagonia: Mallín Pollux. Coyhaique, Province Aysén (45 $41^{\prime} 30^{\prime \prime} \mathrm{S}$, $71^{\circ} 50^{\prime} 30^{\prime \prime} \mathrm{W}, 640 \mathrm{~m}$ elevation). Palaeogeography, Palaeoclimatology, Palaeoecology, 254: 492-507.

MARTINIC, M. 2005. De la Trapananda al Aysén. Pehuén Editores. Santiago, Chile.

MCWETHY, D., C. MCWHITLOCK, J. WILMSHURST, M. MCGLONE, M. FROMONT, X. LI, A. DIEFFENBACHER-KRALL, W. HOBBS, S. FRITZ \& E. COOK. 2010. Rapid landscape transformation in South Island, New Zealand following initial Polynesian settlement. Proceedings of the National Academy of Sciences USA, 107: 21343-21348.

MOSER, K., J. SMOL, G. MACDONALD \& C. LARSEN. 2002. $19^{\text {th }}$ century eutrophication of a remote boreal lake: a consequence of climate warming? Journal of Paleolimnoly, 28: 269-281.

PATERSON, A., B. CUMMING, J. SMOL, J. BLAIS \& R. FRANCE. 1998. Assessment of the effects of logging, forest fires and drought on lakes in northwestern Ontario: a 30-year paleolimnological perspective. Canadian journal of forest Research, 28: 1546-1556.

PATERSON, A., D. MORIMOTO, B. CUMMING, P. SMOL \& J. SZEICZ. 2002. A paleolimnological investigation of the effects of forest fire on lake water quality in northwestern Ontario over the past ca. 150 years. Canadian Journal of Botany, 80: 1329-1336. 
PHILIBERT, A., Y. PRAIRIE \& C. CARCAILLET. 2003a. 1200 years of fire impact on biogeochemistry as inferred from high resolution diatom analysis in a kettle lake from the Picea marianamoss domain (Quebec, Canada). Journal of Paleolimnology, 30: 167-181.

PHILIBERT, A., Y. PRAIRIE, I. CAMPBELL \& L. LAIRD. 2003b. Effects of late Holocene wildfires on diatom assemblages in Christina Lake, Alberta, Canada. Canadian Journal Forest Research, 33: 2405-2415.

QUINTANILLA, V. 2005. Estado de recuperación del bosque nativo en una cuenca nordpatagónica de Chile, perturbada por grandes fuegos acaecidos 50 años atrás $\left(44^{\circ}-45^{\circ} \mathrm{S}\right)$. Revista de Geografía Norte Grande, 34: 73-92.

RIGNOT, E., A. RIVERA \& G. CASASSA. 2003. Contribution of the Patagonia icefields of South America to sea level rise. Science, 302: 434-437.

ROSÉN, P. \& D. HAMMARLUND. 2007. Effects of climate, fire and vegetation development on Holocene changes in total organic carbon concentration in three boreal forest lakes in northern Sweden. Biogeosciences, 4: 975-984.

RUMRICH, U., H. LANGE-BERTALOT \& M. RUMRICH. 2000. Diatomeen der Anden: Von Venezuela bis Patagonien/Feuerland und zwei weitere Beitráge. Iconographia Diatomológica, Volume 9. Koeltz scientific Books, Koenigstein, Germany.

SAROS, J., T. MICHEL, S. INTERLANDI \& P. WOLFE. 2005. Resource requirements of Asterionella formosa and Fragilaria crotonensis in oligotrophic alpine lakes: Implications for recent phytoplankton community reorganizations. Canadian Journal of Fisheries Aquatic Sciences, 62: 1681-1689.

SCHELSKE, C., C. DONAR \& E. STOERMER. 1999. A test of paleolimnologic proxies for the planktonic/benthic ratio of microfossil diatoms in
Lake Apopka. In Proceedings of the 14th International Diatom Symposium, ed. S. Mayama, M. Idei, \& I. Koizumi. Konigstein: Koeltz Scientific Books. Koenigstein, Germany.

SCHINDLER, D. W. 1980. The effect of fertilization with phosphorus and nitrogen versus phosphorus alone on eutrophication of experiment lakes. Limnology and Oceanography, 25: 1149-1152.

SMITH, V., S. JOYE \& R. HOWARTH. 2006. Eutrophication of freshwater and marine ecosystems. Limnology and Oceanography, 51: 351-355.

SZEICZ, J., Y. ZEEB, K. BENNETT \& J. SMOL. 1998. Highresolution paleoecological analysis of recent disturbance in a southern Chilean Nothofagus forest. Journal of Paleolimnology, 20: 235252.

TEMPORETTI, F. 2006. Efecto a largo plazo de los incendios forestales en la calidad del agua de dos arroyos en la sub-región Andino-Patagónica, Argentina. Ecología Austral, 16:157-166.

THEISSEN, K., W. HOBBS, J. HOBBS, K. ZIMMER, L. DOMINE, J. COTNER \& S. SUGITA. 2012. The altered ecology of Lake Christina: A record of regime shifts, land-use change, and management from a temperate shallow lake. Science of the Total Environment, 433: 336-346.

WATCHORN, M., P. HAMILTON, T. ANDERSON, H. ROE \& R. PATTERSON. 2008. Diatoms and pollen as indicators of water quality and land use change: a case study from the Oak Ridges Moraine, Southern Ontario. Journal of Paleolimnology, 39: 491-509.

WHITLOCK, C. \& S. MILLSPAUGH. 1996. Testing the assumptions of fire-history studies: an examination of modern charcoal accumulation in Yellowstone National Park, USA. Holocene, 6: 7-15.

WHITLOCK, C. 2001. Variations in Holocene fire frequency: a view from the western United States. Biology and Environment: Proceedings of the Royal Irish Academy, 101B: 65-77. 